\title{
A CHARACTERISATION OF VON NEUMANN REGULAR JORDAN TRIPLE SYSTEMS
}

\author{
KURT MEYBERG
}

ABSTRACT. In this note we give a characterisation of the Jacobson radical of a Jordan triple system in terms of principal inner ideals. If $(\mathscr{U}, P)$ is a Jordan triple system and Rad $\mathscr{t}$ the Jacobson radical of $\mathscr{U}$ then $x \in \operatorname{Rad} थ$ iff $P(x) थ=P(x+P(x) y)$ U for all $y \in थ$. We use this to give a new characterisation of von Neumann regular Jordan triple systems. In particular, this gives another most elementary and short proof that semisimple Jordan triple systems with dcc on principal inner ideals are von Neumann regular.

Let $\Phi$ be a commutative ring with unit element 1 , $\mathcal{U}$ a unital $\Phi$-module and $P:$ U $\rightarrow$ End $_{\Phi}$ Ul a quadratic map, i.e. $P(\alpha x)=\alpha^{2} P(x)$ for all $\alpha \in \Phi$, $x \in \mathfrak{N}$ and $P(x, y):=P(x+y)-P(x)-P(y)$ is bilinear. We define $L: \mathfrak{U} \times \mathfrak{U} \rightarrow$ End $_{\Phi}$ U⿱ by $L(x, y) z=P(x, z) y$. The pair (Û, P) is a Jordan triple system (=: Jts), if

$$
\begin{gathered}
L(P(x) y, y)=L(x, P(y) x) \\
L(x, y) P(x)=P(x) L(y, x) \\
P(P(x) y)=P(x) P(y) P(x)
\end{gathered}
$$

hold in 2 and all scalar extensions of 2 .

Any quadratic Jordan algebra (unital or not) is a Jts. In Jordan triple systems (as well as in Jordan algebras) the mappings

$$
B(x, y):=\operatorname{Id}-L(x, y)+P(x) P(y)
$$

play an important role. An easy consequence of (1)-(3) is

$$
P(x-P(x) y)=P(x) B(y, x)=B(x, y) P(x) \text {. }
$$

For all formulas and properties of Jts we refer to [2]. If $\mathrm{Rad}$ Q $\mathrm{U}$ denotes the Jacobson radical of the Jts (U, $P$ ) then it is known (see also [1] for Jordan algebras ) that

Received by the editors February 5, 1974. $16 A 30$.

AMS (MOS) subject classifications (1970). Primary 17C10, 17D05, 16A21,

Key words and phrases. Jordan algebra, Jordan triple system, Jacobson radical, von Neumann regular. 


$$
\operatorname{Rad} \mathfrak{U}=\{x \in \mathfrak{U} ; B(x, y) \text { invertible for all } y \in \mathfrak{U}\} \text {. }
$$

The purpose of this note is to add another characterisation of $\operatorname{Rad} \mathscr{U}$ to the known ones and to apply this to von Neumann regularity.

Theorem 1. $B(x, y)$ is invertible iff $P(x) \mathfrak{U}=P(x-P(x) y) \mathfrak{U}$.

Proof. If $B(x, y)$ is invertible, then $B(y, x)$ is invertible (by the symmetry principle [2]). Then $B(y, x) \mathfrak{U}=\mathscr{U}$ and (4) implies

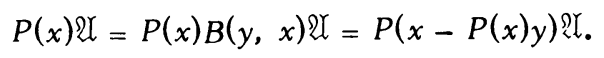

If conversely, $P(x) \mathfrak{U}=P(x-P(x) y)^{\mathscr{U}}=B(x, y) P(x) \mathfrak{U}$, then

$$
P(x) \mathfrak{U} \subseteq B(x, y) \mathfrak{U},
$$

and (by definition)

$$
\begin{aligned}
B(x, y) x & =x-2 P(x) y+P(x) P(y) x, \\
& =x+P(x) w, \text { for some } w \in \mathfrak{U}, \\
& =x+B(x, y) u, \text { for some } u \in \mathfrak{U} \text { (by (6)). }
\end{aligned}
$$

Thus $2 x \in B(x, y)^{\mathscr{U}}$. This together with (6) gives $2 x-P(x) y \in B(x, y)^{\mathscr{U}}$, and consequently $B(x, y)$ is invertible [2, p. 137].

Theorem 2. (a) $\operatorname{Rad} \mathfrak{U}=\{x \in \mathfrak{U} ; P(x) \mathfrak{U}=P(x-P(x) y) \mathfrak{U}$ for all $y \in \mathfrak{U}\}$. (b) $\operatorname{Rad} \mathfrak{U}=\{x \in \mathfrak{U} ; P(y) \mathfrak{U}=P(y-P(y) x) \mathfrak{U}$ for all $y \in \mathfrak{U}\}$.

Part (a) is an immediate consequence of (5) and Theorem 1; part (b) follows from (a) and the symmetry principle.

We recall, an element $x \in \mathfrak{U}$ is von Neumann regular if $x \in P(x) \mathscr{U}$. $\mathscr{U}$ is von Neumann regular if all elements in $\mathscr{O}$ are von Neumann regular.

Theorem 3. A Jordan triple system $\mathfrak{U}$ is von Neumann regular if and only if $\mathrm{Rad} \mathscr{U}=0$ and in any strictly descending infinite chain of principal inner ideals

$$
P\left(x_{1}\right) \mathfrak{U} \supsetneqq P\left(x_{2}\right) \mathfrak{N} \supsetneqq \cdots \supsetneqq P\left(x_{m}\right) \mathfrak{U} \supsetneqq \cdots
$$

there is a $k$ such that $x_{k}$ is von Neumann regular.

Corollary. If $\mathfrak{U}$ is a Jts with dcc on principal inner ideals, then $\mathscr{U}$ is von Neumann regular iff $\mathrm{Rad} \mathfrak{X}=0$.

Remark. The corollary (with a completely different proof) is already in [3].

Proof. If $\mathscr{U}$ is von Neumann regular, then $\operatorname{Rad} \mathscr{O}=0$ and in any principal inner ideal $P(x)$ OC the generating element $x$ is von Neumann regular.

Conversely, assume $\mathrm{Rad} \mathfrak{U}=0, x \in \mathcal{U}, x \neq 0$. Then by Theorem 2(a) there is $y \in \mathfrak{U}$ such that $P(x) \mathfrak{U}, \neq P(x-P(x) y)$ U. Then $P(x) \mathfrak{U} \supsetneqq$ 
$P(x-P(x) y)$ \& (again (4))。Now, if $x_{1}:=x-P(x) y$ is not von Neumann regular, we continue. There is $y_{1} \in \mathcal{U}$ such that $P\left(x_{1}\right) \mathfrak{U} \supsetneqq P\left(x_{2}\right)$ Ul where $x_{2}=x_{1}-P\left(x_{1}\right) y_{1}$. In this way we construct a strictly descending chain of inner ideals

$$
\begin{gathered}
P\left(x_{0}\right) \mathfrak{A} \supsetneqq P\left(x_{1}\right) \mathfrak{Q} \supsetneqq P\left(x_{2}\right) \mathfrak{Q} \supsetneqq \cdots, \\
x_{k+1}:=x_{k}-P\left(x_{k}\right) y_{k}, \quad x_{0}=x, \quad y_{0}=y .
\end{gathered}
$$

If this chain terminates at $P\left(x_{n}\right) \mathcal{Q}=0$, then $x_{n}=0$ (and is $\mathrm{v}$ on Neumann regular), since $\mathrm{Rad} \mathscr{U}=0$ implies that there are no trivial elements (= absolute zero divisors) different from 0. (This case always occurs if 0 has dcc on principal inner ideals.) If the chain is infinite, then by assumption we reach a von Neumann regular element, say $x_{s}$. Now, let $l$ be the least number such that $x_{l}$ (as constructed above) is von Neumann regular. If $l \geq 1$, then it follows from McCoy's lemma [3] and $x_{l}=x_{l-1}-P\left(x_{l-1}\right) y_{l-1}$ that $x_{l-1}$ is regular, a contradiction. Consequently $l=0$ and $x=x_{0}$ is von Neumann regular.

Remark. The same results hold in associative rings, since any associative (or alternative) ring together with $y \mapsto x y x$ is a Jts. Of course, in this case the proofs are much simpler than for arbitrary Jts. But for rings we have also the linear version of the above results. The characterisation of the radical is certainly well known. It is similar to a characterisation given by Szász in [4]. If $R$ is an associative ring, then $x \in \operatorname{Rad} R$ iff $x R=(x-x y x) R$ for all $y \in R$. If $x \in \operatorname{Rad} R$, then $1-y x$ is invertible (assume for simplicity $1 \in R)$ and then $x R=(x-x y x) R$. If conversely, $x R=(x-x y x) R$, then $x y=$ $x u-x y x u$ for some $u$, which shows that $x y$ is right quasi invertible. If this holds for all $y \in R$, then $x \in \operatorname{Rad} R$. The corresponding chain condition (as in Theorem 3 ) is for principal right ideals. $R$ is von Neumann regular iff $\operatorname{Rad} R=0$ and any infinite strictly descending chain of principal right ideals contains a direct summand. These results are left and right symmetric.

\section{REFERENCES}

1. K. McCrimmon, A characterisation of the radical of a Jordan algebra, J. Algebra 18 (1971), 103-111. MR 43 \#3316.

2. K. Meyberg, Lectures on algebras and triple systems, Lecture notes, University of Virginia, Charlottesville, Va., 1972.

3. $\longrightarrow$, Von Neumann regularity in Jordan triple systems, Arch. Math. 23 (1972), 589-593.

4. F. Szász, Äquivalenzrelation für die Charakterisierung des Jacobsonschen Radikals, Acta Math. Acad. Sci. Hungar 22 (1971/72), 85-86. MR 45 \#8674.

MATHEMATISCHES INSTITUT DER TUM, D-8000 MÜNCHEN (MUNICH) 2, POSTFACH 202420, FEDERAL REPUBLIC OF GERMANY 